\title{
The Jungle of Methods for Evaluating Phenotypic and Phylogenetic Structure of Communities
}

Author(s) :Juli G. Pausas and Miguel Verdú

Source: BioScience, 60(8):614-625. 2010.

Published By: American Institute of Biological Sciences

URL: http://www.bioone.org/doi/full/10.1525/bio.2010.60.8.7

BioOne (www.bioone.org) is a nonprofit, online aggregation of core research in the biological, ecological, and environmental sciences. BioOne provides a sustainable online platform for over 170 journals and books published by nonprofit societies, associations, museums, institutions, and presses.

Your use of this PDF, the BioOne Web site, and all posted and associated content indicates your acceptance of BioOne's Terms of Use, available at www.bioone.org/page/terms_of_use.

Usage of BioOne content is strictly limited to personal, educational, and non-commercial use. Commercial inquiries or rights and permissions requests should be directed to the individual publisher as copyright holder.

BioOne sees sustainable scholarly publishing as an inherently collaborative enterprise connecting authors, nonprofit publishers, academic institutions, research libraries, and research funders in the common goal of maximizing access to critical research. 


\title{
The Jungle of Methods for Evaluating Phenotypic and Phylogenetic Structure of Communities
}

\author{
JULI G. PAUSAS AND MIGUEL VERDÚ
}

\begin{abstract}
The way communities are assembled is an old ecological question currently experiencing renewed interest thanks to the recent advances in molecular biology and phylogenetics. The generality of these new methods has allowed us to understand the structure of communities of organisms from different kingdoms and at different scales. Concomitant with this growing interest, new methods, metrics, terms, and software have appeared that independently solve similar questions, but with different approaches. Here we provide a unifying framework on methods for community structure based on the relationships between four key concepts: phylogeny, phenotype, environment, and co-occurrence. The different approaches are based on different community representations of traits, the phylogenetic relationships of species in the community, or species occurrence along the environmental gradients. We finally provide insights on future directions of this emerging discipline.
\end{abstract}

Keywords: community structure, phylogenetic pattern, phenotypic pattern, clustering, overdispersion

M any people wonder why so many desert plant communities are dominated by spiny species, most of them cacti. The first observation refers to the community's phenotypic structure and the second to its phylogenetic structure. What do these observations tell us about the mechanisms assembling these communities?

The structure of ecological communities is driven by biogeographical and ecological processes. Large-scale biogeographical processes determine the regional species pool from which ecological communities are assembled. In turn, ecological processes at local scales may give feedback to influence the composition and diversity of the regional pool. The two main processes traditionally thought to structure ecological communities are competition and habitat filtering (e.g., Weiher and Keddy 1995, Wilson et al. 1999). Other key community processes, such as those related to food-web interactions, have also been considered (e.g., Paine's classic experiments in intertidal communities, the Janzen-Connell hypothesis in tropical forest). With the increasing availability of phylogenetic information there has been a revival of interest in understanding the evolutionary dimensions of local community assembly processes. In this context, Webb and colleagues (2002) provided a novel framework in which phylogenetic information from co-occurring species is used as an indicator of the two main assembly processes (competition and habitat filtering), although other ecological processes considering food-web interactions are increasingly examined. Since Webb and col- leagues' (2002) seminal paper there has been a bloom of methods aimed at testing the structure of communities. Each of these methods is based on different approaches, such as trait similarity, phylogenetic distances, and species co-occurrence. Accordingly, a plethora of metrics, terms (see box 1), null models, statistical tests, and computer programs (table 1) are overwhelming newcomers to this developing research field. Here we show that this apparent complexity can be framed using a unifying methodological scheme based on the relationships between phylogeny, phenotype, environment, and cooccurrence (figure 1). We start by briefly tracing the foundations of current community structure methods, and then review these approaches in light of the proposed unifying framework. Finally, we provide insights on new, developing ideas.

\section{Classic approaches}

"As species of the same genus have usually, though by no means invariably, some similarity in habits and constitution, and always in structure, the struggle will generally be more severe between species of the same genus, when they come into competition with each other, than between species of distinct genera" (Darwin 1859, p. 76). Following Darwin's influential view that competition is the main driver of evolution, the study of community assembly processes focused on how competitive exclusion limits the ecological similarity of co-occurring species. This view was fostered by the Lotka and Volterra mathematical models of competition, and by Gause's 


\begin{tabular}{|lll|}
\hline Table 1. Available software for community structure analysis. & Use \\
\hline Software name & Availability (Web address) & 1 in figure 1 \\
\hline EcoSim & www.garyentsminger.com/ecosim, http://cran.r-project.org/web/packages/picante & 2.1 in figure 1 \\
TraitHull & www.pricklysoft.org/software/traithull.html & 2.2 in figure 1 , box 3 \\
Ape & ape.mpl.ird.fr, cran.r-project.org/web/packages/ape & 3.2 in figure 1 \\
PhySig (MatLab scripts) & www.biology.ucr.edu/people/faculty/Garland/PHYS/G.html, http://cran.r-project.org/web/ & \\
& packages/picante & 3.2 in figure 1 \\
BayesTraits & www.evolution.reading.ac.uk/BayesTraits.html & 3.2 in figure 1 \\
PDAP & www.biology.ucr.edu/people/faculty/Garland/PDAP.html & 3.1 and 3.2 in figure 1, box 3 \\
Phylocom & www.phylodiversity.net/phylocom, http://cran.r-project.org/web/packages/picante & 3.1 in figure 1, box 2 \\
MatLab scripts & Supplementary material in Helmus and colleagues (2007b), http://cran.r-project.org/ \\
SpaCoDi & web/packages/picante & 3.1 in figure 1 , box 2 \\
EcoPhyl & www.ulb.ac.be/sciences/bioancel/ohardy/,http://cran.r-project.org/web/packages/picante & 3.1 in figure 1 , box 2 \\
MatLab scripts & www.cbs.umn.edu/cavender/ & 4 in figure 1 \\
Geiger & As supplementary material in Helmus and colleagues (2007a) & Box 3 \\
\hline
\end{tabular}

classical competitive exclusion experiments. These studies suggested that when species compete for the same limiting resource, all but one species will be driven to extinction. On the other hand, species can coexist by inhabiting different niches that partition the available resources. The concept of limiting similarity was an outgrowth of the competitive exclusion principle, which suggests that similar species cannot coexist; thus, there is a limit to the number of species inhabiting a particular community, at which niche space is fully saturated. MacArthur and Levins (1967) evaluated the limit of the degree of similarity allowing for coexistence using a model that linearly ordered the resource-consuming species along a resource axis. Diamond (1975) presented an influential paper setting a number of community assembly rules that predict how competition leads to a nonrandom species co-occurrence pattern (1 in figure 1). These rules became controversial after the application of appropriate null models (Connor and Simberloff 1979, Gotelli and Graves 1996, Ulrich 2004), and what followed was a massive debate about community structure, mainly focused on searching for empirical evidence that competition structured communities (see Weiher and Keddy 1999). However, a number of studies demonstrated that two or more competitors could coexist while limited by a single resource, questioning the universality of limiting similarity. Indeed, coexistence of similar species can be driven by other processes, such as the spatial and temporal heterogeneity of resources and environmental conditions, or

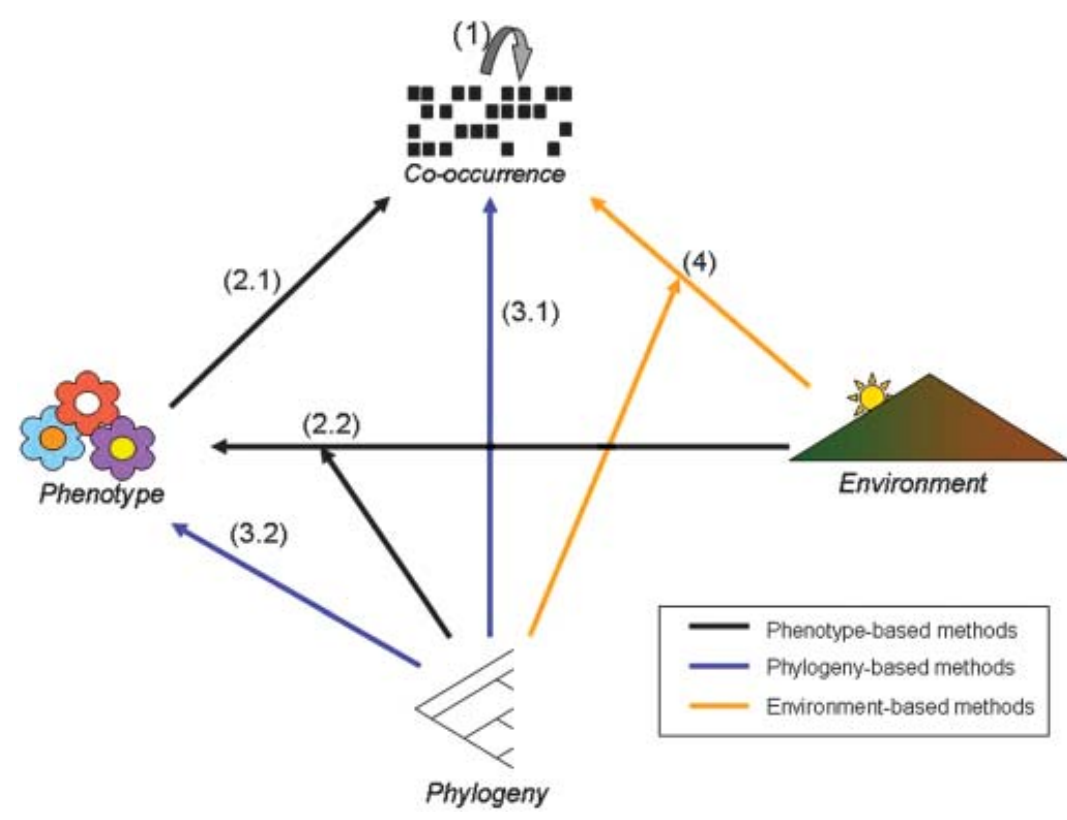

Figure 1. Methods for analyzing community structure can be represented in a simple framework in which the relationships (arrows) between the four key concepts (phylogeny, phenotype, environment, and co-occurrence) are integrated. Bracketed numbers refer to (1) the co-occurrence pattern versus the random expectation. Phenotype-based approach: (2.1) the relationship between the species' phenotypes and their co-occurrence (phenotypic community structure); (2.2) the relationship between species' response to the environment and the species' phenotypes, controlling by the species' phylogenetic relatedness (phenotypic community structure); (3) the phylogeny-based approach; (3.1) the relationship between the species' phylogenetic relationships and their co-occurrence (phylogenetic community structure); (3.2) the relationship between the species' phenotypes and their phylogenetic relationships (trait evolution); (4) the environment-based approach: the relationship between species' response to the environment and the co-occurrence, considering the phylogenetic relatedness (phylogenetic community structure). 
the occurrence of greater intra- versus interspecific competition (Abrams 1983, Tilman 1985, Chesson and Kuang 2008).

Another methodological criticism of the community assembly rules was the simplicity of the niche overlap measures between species. Ricklefs and Travis (1980) proposed an alternative approach on the basis of morphological similarities among species in a multidimensional space (i.e., morphospace); their premise was that "the adaptations of organisms reflect their ecological relationships, specifically that morphological space can be mapped closely onto ecological space" (Ricklefs and Travis 1980, p. 322). Ricklefs and Travis characterized community structure by estimating the total morphospace volume occupied by each community and the distances between species in the morphospace. In this framework, competition is indicated by a standard deviation (SD) of nearest-neighbor distances along trait axes within the community that are lower than those predicted by the null model (2.1 in figure 1; Ricklefs and Travis 1980).

Whereas previous models were based on competition, other attempts to understand community structure considered the community composition to be the outcome of environmental factors filtering species with particular phenotypes. In this context, the environment is viewed as a filter, removing species that lack traits for persisting under a particular set of conditions (Keddy 1992). Examples include whether a species can germinate underwater in wetland communities (van der Valk 1981) or under different fire regimes (Noble and Slatyer 1980).

Many researchers realized that the taxonomic hierarchy indicates relatedness among taxa and therefore provides clues to phenotypic similarity. After this finding, species-to-genus ratios were used as a metric in community structure studies (e.g., Simberloff 1970). Recent approaches incorporate this rationale by enhancing the accuracy of the information on species relatedness from phylogenetic information.

\section{Recent approaches}

The incorporation of phylogenetics to the classical approaches has laid the foundation of the emerging research area of community phylogenetics, and has been the impetus for the development of many tools for detecting the underlying force structuring communities (see reviews in Webb et al. 2002, Cavender-Bares et al. 2009, Vamosi et al. 2009). The objective of the different methods and approaches is to detect patterns (e.g., clustering, overdispersion) from which it is possible to infer community processes (e.g., filtering, competition). The different approaches may be based on the different community representations of traits (phenotype-based methods), the phylogenetic relationships of the species in the community (phylogeny-based methods), or the species occurrence along environmental gradients (environment-based methods; figure 1). The appropriate use of these methods requires an explicit definition of the taxonomical and biogeographical scales, because by increasing the size of the spatial context we move the emphasis from local to biogeographical processes. Several studies have shown that increasing the spatial or taxonomic scale of the reference species pool leads to greater environmental heterogeneity, and consequently to a higher relative importance of habitat filtering in explaining community structure (Cavender-Bares et al. 2006, 2009, Swenson et al. 2006, Kraft et al. 2007, Emerson and Gillespie 2008, Graham and Fine 2008, Vamosi et al. 2009). This is because the communities are considered open entities (following the metacommunity approach) and are linked by the dispersal of multiple potentially interacting species (Leibold et al. 2004).

Phenotype-based methods. The aim of phenotype-based methods is to infer the assembly process from the distribution of species traits in the communities relative to the distribution of traits in the available species pool. Such distribution corresponds to the phenotypic community structure, with two contrasted patterns: phenotypic clustering and phenotypic overdispersion (figure 2). Phenotypic clustering arises when co-occurring species are more similar (phenotypically) than expected from the distribution of traits in the regional species pool, whereas phenotypic overdispersion refers to cooccurring species that are less similar (phenotypically) than expected in the same species pool (see box 1). In general, environmental filtering leads to phenotypic clustering, whereas competition leads to phenotypic overdispersion (Weiher and Keddy 1995). We note, however, that if there is substantial environmental heterogeneity within the area of study, then different microenvironments may filter different traits, leading to an overall lack of phenotypic clustering.

The phenotypic structure of the community can be detected by studying the phenotypes of co-occurring species (2.1 in figure 1). When competition is the main assembly process, phenotypically similar species tend to exclude each other, generating evenly distributed trait values along community trait axes. Such trait representation in the community can be detected when the standard deviation of nearest-neighbor distance measured along trait axes is lower (species are spaced more evenly), or when the kurtosis of the distribution of trait values is smaller than the expected value in randomly assembled communities from the regional pool (figure 2; Ricklefs and Travis 1980, Kraft et al. 2008, Cornwell and Ackerly 2009, Ingram and Shurin 2009). Using this approach, Rabosky and colleagues (2007) suggested that competition is the main driving force leading to the overdispersion of body size in Australian lizard communities. Another way to test whether dissimilar phenotypes co-occur is by correlating phenotype distances and co-occurrence matrices. Such a method enabled Cavender-Bares and colleagues (2004) to detect phenotypic overdispersion in a set of Floridian oak traits such as acorn maturation time, freezing embolism, leaf life span, and first-year vessel diameter.

When environmental filtering is the main assembly process, the resulting co-occurring species will necessarily have the traits conferring the ability to tolerate such filtering. Consequently, the co-occurring species will be phenotypically similar (phenotypic clustering; figure 2). The matrix correlation method (co-occurrence versus phenotype) provides evidence that plants species sharing the same postfire germination trait tend 
a

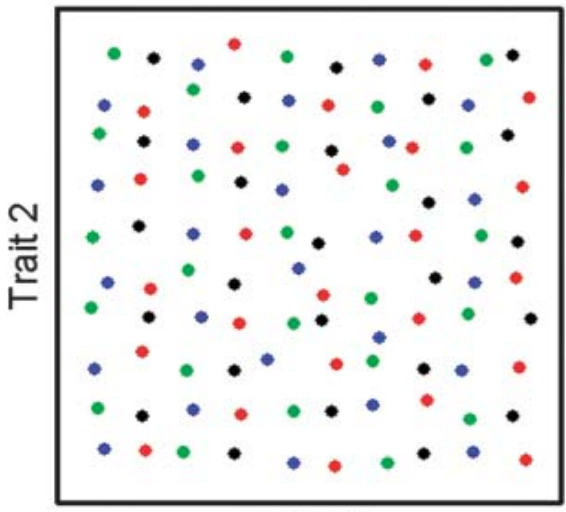

Trait 1

NNsd $=0.017$ b

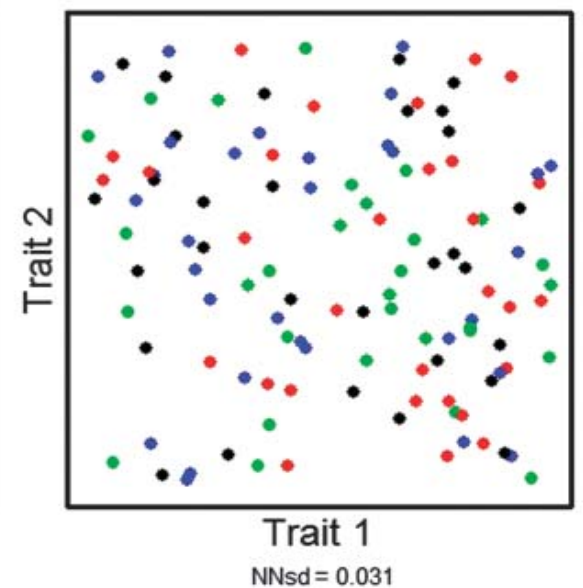

c

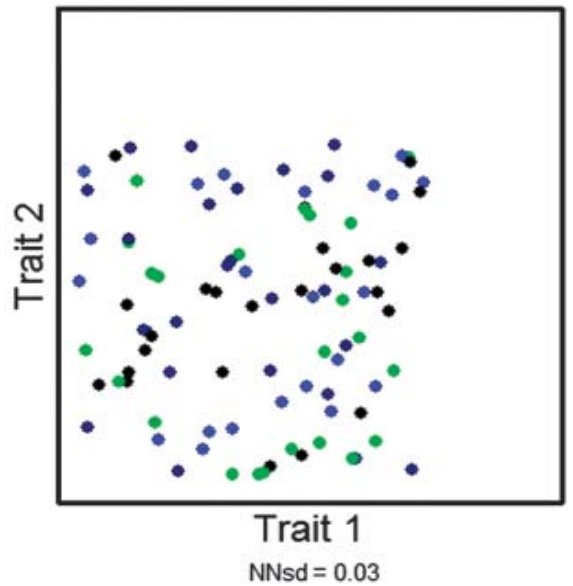

Figure 2. Graphical example of (a) phenotypically overdispersed, (b) random, and (c) clustered communities. Each point represents a species in the morphospace determined by three noncorrelated traits, two quantitative traits ( $\mathrm{x}$-and $\mathrm{y}$-axes), and a qualitative trait (symbol color). Overdispersed communities have the lowest standard deviation of the nearest-neighbor distance (NNsd), whereas clustered communities have a reduced range of trait values (i.e., reduced trait space occupied the species, plot c).

Box 1. The jargon jungle.

In spatial ecology (i.e., the study of the spatial structure of sessile organisms), three concepts are used to describe the spatial dispersion of organisms (Perry et al. 2002). When the locations of organisms are independent of one another, so that all locations have the same probability of containing an organism (null model of dispersion), it is considered a random pattern. When the presence of one organism increases the probability of finding another in the vicinity, then it is said that there is species attraction, and the pattern has many names, including clumped, clustered, underdispersed, contagious, aggregated, or patchy; all referring to the same concept. Finally, when the presence of an organism reduces the probability of finding another nearby, it is said that there is species repulsion, and the pattern is typically called overdispersed, evenly dispersed, or described as having a regular, uniform, or checkerboard pattern. However, the term overdispersed has also been used to refer to clumped patterns (e.g., Connor et al. 1997); this is because in statistical terms, overdispersion is used when the variance is greater than the mean in a frequency distribution (overdispersed data). Thus, to avoid ambiguities, the use of the term overdispersion has been discouraged (Perry et al. 2002). There are many methods to test the deviation from the random pattern based on the (spatial) distance between the studied organisms (e.g., neighbor distance methods, individual-to-all-individual distance methods, etc.).

These concepts have been adopted in community ecology; they use the phenotypic distances when studying trait-state dispersion, and the phylogenetic distances when studying the phylogenetic community structure. For instance, phenotypic clustering (i.e., phenotypic underdispersion) means that the co-occurring species are more phenotypically similar (species attraction) than what we would expect under the null model (i.e., all phenotypes having the probability given by the regional species pool). Phylogenetic overdispersion (i.e., phylogenetic repulsion) means that the co-occurring species are less closely related than expected by chance (i.e., as it would be expected for the phylogeny of the regional species pool). In the jargon of community phylogenetics, clustering and overdispersion are perhaps the most commonly used terms to refer to the two contrasting patterns (Webb et al. 2002), although some authors use phylogenetic evenness instead of overdispersion to avoid the ambiguities mentioned above. Another way to escape this jargon jungle is to talk about high and low dispersion, referring to higher and lower dispersion than random, respectively. In this article, we use the original terminology used in the seminal paper by Webb and colleagues (2002): clustering and overdispersion.

to co-occur (phenotypic clustering) in fire-prone communities (Verdú and Pausas 2007). Using the trait variability approach, the clearest signal of phenotypic clustering produced by environmental filtering is a reduction in the range of trait values from the one expected from the regional species pool (Cornwell et al. 2006, Kraft et al. 2008, Ingram and Shurin 2009). For instance, in Amazonian forests, functional traits such as specific leaf area (SLA), leaf nitrogen concentration, and maximum tree size showed a reduced range of values compared with the null model generated from the regional pool, clearly indicating phenotypic clustering (Kraft et al. 2008). Although these methods reveal overall patterns at the community level, it is also possible to detect the relative contribution of each trait involved in the environmental filtering of species from specific clades in specific environments (Mayfield et al. 2009).

Once phenotypic clustering is detected, it is important to determine the environmental factor filtering the phenotypes. For instance, in the above Amazonian example, ridge tops tend to be composed of species with lower SLA (i.e., high sclerophylly) and smaller leaves compared with valley communities. To infer a causal relationship between phenotypes and environment, one must take the phylogenetic relatedness among species into account to control for the pseudoreplication derived from common ancestry. Cavender-Bares and 
colleagues (2004) used phylogenetic independent contrasts to correlate functional traits (such as transpiration rate) with habitat (soil moisture) preferences in Floridian oaks. Alternatively, this phylogenetically controlled correlation may be performed by means of partial Mantel tests among the corresponding distance matrices (Elias et al. 2008), or by ordination methods that account for environment, traits, and species (e.g., RLQ analysis; Dray and Legendre 2008). Ferrier (2007) recently proposed a more general test for matrix correlations that allows the detection of nonlinear responses to the environment. All of these methods have the drawback that a single environmental value (typically the mean) is assigned for each species without considering the difference in species' environmental ranges. This can be overcome by using recent comparative methods that incorporate intraspecific variability in the analysis (e.g., Felsenstein 2008).

To account for the multidimensional nature of phenotypes, an early approach was to estimate species clustering using the minimum spanning tree, which is the shortest path connecting the species in the trait space (e.g., Ricklefs and Travis 1980). Cornwell and colleagues (2006) developed a similar trait-based approach using the idea of the multidimensional niche (Hutchinson 1957) and community hypervolume (Ricklefs and Travis 1980). Cornwell and colleagues quantified the trait space (morphospace) occupied by a community using the convex hull volume- that is, the smallest convex contour enclosing the points (species) in the trait spaceand compared it with a null model of a randomly assembled community with the same species richness. Filtering is demonstrated by the reduction of the volume, indicating that the range of successful combination of traits in the community is restricted. A clear example is how recurrent fires, by precluding the entry of some phenotypes into community, reduce the trait combination and community volume (Pausas and Verdú 2008). Although this method was designed for testing habitat filtering, it could be combined with a test of competition (see "Going beyond the dichotomy," below).

Phylogeny-based methods. The aim of phylogeny-based methods is to infer the assembly process from the phylogenetic relationships of the co-occurring species in the communities. Indeed, phylogenetic relatedness can be considered a proxy for the multidimensional phenotypes, given that phenotypes are likely to be conserved because of common ancestry. Phylogenetic clustering and overdispersion are the two contrasted phylogenetic community structures; the former is indicated by closely related species co-occurring more often than expected by the null model, the latter by closely related species co-occurring less often than expected (3.1 in figure 1).

Webb and colleagues (2002) combined the phylogenetic and phenotypic species information from the community to differentiate between environmental filtering and competitive exclusion. Because environmental filtering allows only species with particular traits to enter the community, coexisting species will possess similar phenotypic characters (i.e., phenotypic clustering). Therefore, the community phy- logenetic structure depends on whether the trait evolved in a conserved or convergent way (figure 3). If closely related species are similar in the trait that confers tolerance (trait conservatism), then coexisting species will not only be phenotypically clustered but also phylogenetically clustered (Com-1 in figure 3). Alternatively, if distantly related species are more similar than expected by chance (trait convergence), then coexisting species will be sampled from different clades and thus phylogenetic overdispersion will characterize the community structure (Com-3 in figure 3 ).

In contrast, competition exclusion refers to the process leading to the coexistence of species with different traits (i.e., phenotypic overdispersion) to avoid niche competition. If the trait is conserved, competition will limit the co-occurrence of phenotypically similar-and therefore phylogenetically closely related-species, generating a phylogenetically overdispersed community (Com-2 in figure 3). When the trait is convergent, competition will limit the co-occurrence of phylogenetically distant species (Com-4 in figure 3 ), which produces different phylogenetic patterns depending on the outcome of the species interactions. For instance, phylogenetic clustering could arise from pair-wise competition; species from one subclade always outcompete those in a different subclade. However, as this situation seems unlikely, randomness is the most expected phylogenetic pattern.

To test for these assembly processes we need to quantify both the trait evolution and the phylogenetic structure of the community. Trait evolution (conservatism and convergence) can be assessed by studying the relationship between phenotypes and phylogenetic distances (3.2 in figure 1). This relationship, known as phylogenetic signal, can be estimated by different methods, including matrix correlation, parsimony step reconstruction, generalized least-squares models, phylogenetic independent contrasts, and the tree geometric pattern (Blomberg et al. 2003, Revell et al. 2008, Stayton 2008). It should be noted that a significant phylogenetic signal is reflecting only statistical dependence among species' trait values, which is the pattern required for testing the assembly processes; however, we cannot infer the evolutionary process behind the pattern depicted by the phylogenetic signal (Revell et al. 2008).

Different metrics have been developed to quantify phylogenetic community structure, and each of theses metrics is sensitive to different aspects of community structure, such as the dominant assembly process or the species pool size (box 2). All these methods are certainly subject to error; the use of a simulation approach will allow quantification of the statistical performance of each method with different scenarios (box 3). It is also important to detect which clades of the phylogenetic tree are the most responsible for the community structure, because apparently random community structures may arise as a consequence of opposite phylogenetic patterns in different clades. In general, these methods test for a different (over- or under-) representation in the communities of species within a particular clade 


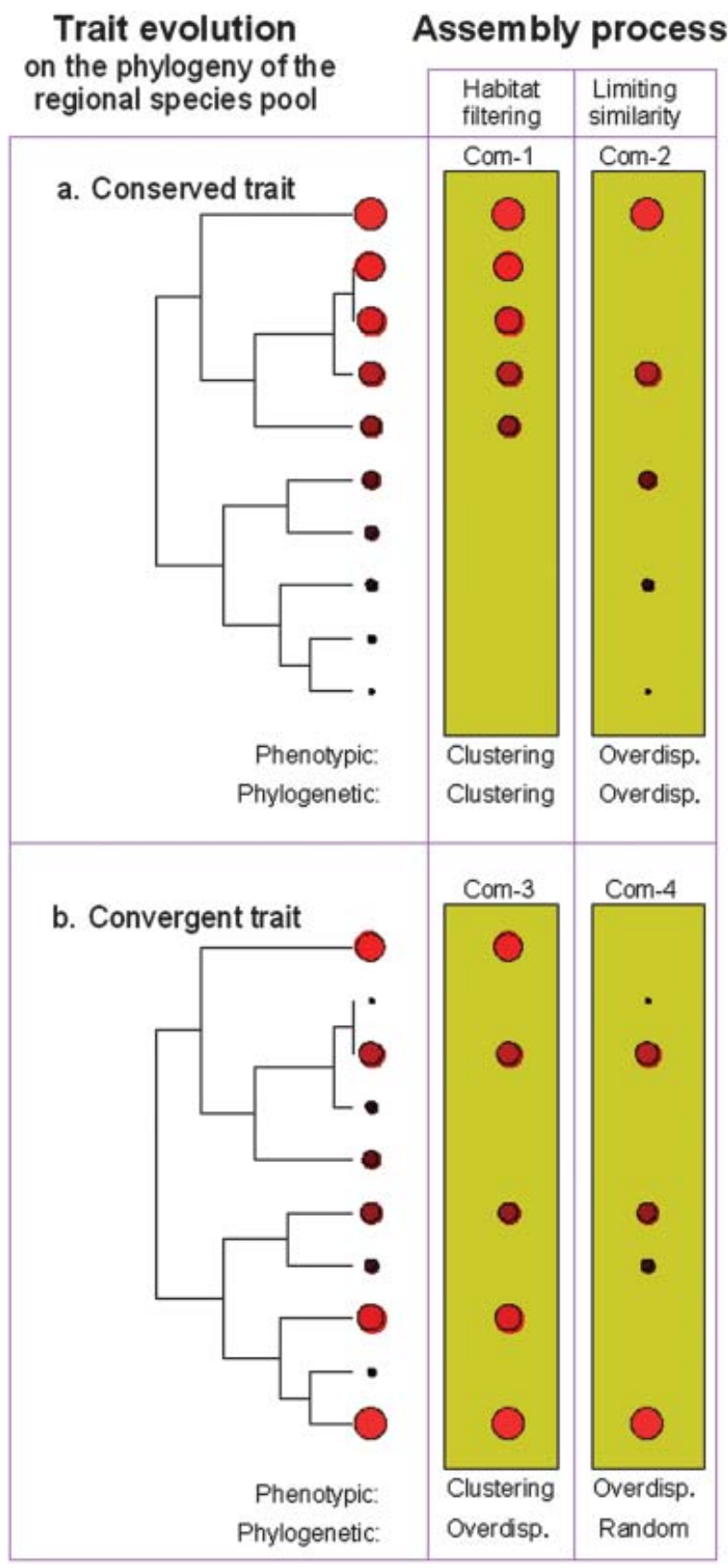

(Cavender-Bares et al. 2004, Hardy and Senterre 2007, Helmus et al. 2007a, Mayfield et al. 2009).

Most of these methods cannot be used to reveal complex phylogenetic patterns at the metacommunity level; for example, different communities can share similar values of clustering without sharing common lineages. By phylogenetically weighting the co-occurence matrix, it is possible to scale up phylogenetic structure toward metacommunities (Pillar and Duarte 2010).

Ultimately, any of the indicators of the phylogenetic structure in the community should be related to the prevalence of particular phenotypes in the community, or to environmental parameters. For instance, in Mediterranean plant communities, phylogenetic clustering was observed in communities
Figure 3. The phenotypic and phylogenetic structure of the community is a consequence of the trait evolution (conserved versus convergent) and the dominant assembly process (habitat filtering versus limiting similarity). Red dots represent a quantitative trait (e.g., seed size, height, specific leaf area, etc.) that can be conserved (a) or convergent (b) in the phylogeny. Green boxes represent four hypothetical communities (Com-1 to Com-4) with five species selected from a regional pool of ten species. Habitat filtering allows the persistence of species with large red dots (Com-1 and Com-3), generating a phenotypic clustering; the phylogenetic structure depends on the trait conservatism. Limiting similarity processes such as competition or facilitation prevent similar species from co-occurring in Com-2 and Com-4, and therefore produce a phenotypic overdispersion (overdisp.). In that case, the phylogenetic structure would be overdispersed for conserved traits, but can be clustered, random, or overdispersed for convergent traits.

under high fire frequency, whereas phylogenetic overdispersion occurred in those subject to low fire frequency (Verdú and Pausas 2007, Ojeda et al. 2010).

Environment-based methods. The aim of the environmentbased methods is to infer the assembly process from the species' response to the environment. This approach follows a similar rationale to that proposed by Webb and colleagues (2002), but considers the species' response to the environment as the focal trait. Indeed, the species' response to the environment can provide clues for the co-occurrence of the species within communities. CavenderBares and colleagues (2004) used the correlation between the species-environmental overlap matrix and phylogenetic distance matrix and found the same results as correlating the co-occurrence and phylogenetic distance matrices.

Helmus and colleagues (2007a) provided a specific method to detect community phylogenetic structure driven by environmental variables ( 4 in figure 1 ). The species' response to the environment is evaluated by regressing species occurrence or abundances against environmental variables. Regression models that do not capture unimodal species' responses in large environmental gradients should be used with caution (e.g., see Huisman et al. 1993 for appropriate regression models). Testing the phylogenetic signal of the regression coefficients produces an indication of habitat filtering. If related species respond similarly to the same environmental factor (conserved response), then phylogenetic clustering through habitat filtering occurs (as in Com-1 in figure 3). Similarly, habitat filtering could also occur when the species' response to environment is similar in distantly related species (convergent response), thus producing a phylogenetic overdispersion of the community (as in Com-3 in figure 3). The latter pattern could be tested by detecting convergent trait evolution of the regression coefficients. 
Webb and colleagues (2002) estimated phylogenetic structure by comparing the mean phylogenetic distance (MPD) or mean nearestneighbor phylogenetic distance (MNND) of the species inhabiting each community against the random expectation based on the regional species pool, in such a way that lower MPD or MNND would indicate phylogenetic clustering, where as higher MPD or MNND would indicate overdispersion (figure 3). The net relatedness index (NRI) and the nearest taxon index (NTI) are standardized forms of MPD and MNND, respectively, and are used for measuring the degree of phylogenetic dispersion (high values indicate clustering whereas low values indicate overdispersion; see Vamosi et al. 2009 for a deep review, with examples). The NTI has a greater power to detect patterns due to competition, whereas the NRI performs better with environmental filtering (Kraft et al. 2007). Regarding the size of the local community relative to the regional species pool, the statistical power increases with pool size for filtered communities but decreases for communities dominated by competition (Kraft et al. 2007). The presence of unresolved nodes, especially in deep nodes, increases type II error (failure to detect nonrandom patterns); indeed, NRI is more sensitive to unresolved nodes than NTI, as the number of nodes needed for computing NRI is in general larger (Swenson 2009). In addition, tests for phylogenetic structure are also sensitive to the phylogenetic signal of both regional species abundance and traits, as well as to the type of null model considered (see Hardy 2008 and Kembel 2009 for performance tests of some of the metrics).

A traditional measure of phylogenetic diversity (PD), calculated as the minimum tree length connecting all the taxa to the root of the tree (Faith 1992), has similarly been used to study the phylogenetic community structure (Proches et al. 2006, Pavoine et al. 2009). Indeed, clustering is indicated by PD values lower than those predicted by the regression between PD and species richness (Forest et al. 2007, Coca and Pausas 2009).

After Webb and colleagues, several alternative phylogeny-based methods to detect community structure have appeared. Helmus and colleagues (2007b) developed another metric, named phylogenetic species variability (PSV), that measures the variance among species of a community in the value of a hypothetical neutral trait evolving under a Brownian motion model. Because phylogenetic relatedness decreases the variance of the hypothetical trait, phylogenetic clustering (overdispersion) would be indicated by a PSV value lower (higher) than expected by chance. Hardy and Senterre (2007) proposed a method based on additive partitioning of the phylogenetic diversity (mean divergence time between species) within and between communities. This method indicates phylogenetic clustering when mean divergence time within community is lower than between communities, and overdispersion otherwise. One of the advantages of this method is that it is less dependent of the regional species pool for detecting community structure (Hardy and Senterre 2007). Yet another way to quantify the community structure is to use the standard deviation of the number of species per phylogenetic node (accounting only for the nodes present in the community species pool), and thus without considering branch lengths (Prinzing et al. 2008). When this approach is used, clustering is indicated by standard deviation values lower than expected by chance.

All of the abovementioned methods are based on comparing phylogenetic relatedness of the species within and between communities. Another category of methods is based on comparing the degree of co-occurrence of species pairs in relation to the phylogenetic distance between them. This correlation must be compared against the appropriate null model. Negative (positive) correlation indicates that closely related species tend to co-occur more (less) often than expected by chance; that is, communities are phylogenetically clustered (overdispersed). Examples of the use of this method can be found in Cavender-Bares and colleagues (2004, 2006) and Helmus and colleagues (2007a). Similarly, phylogenetic community structure can be tested by computing the phylogenetic signal of an occupancy measure (e.g., occurrence, abundance; Cadotte et al. 2009).

Many of the above methods (e.g., MPD and MNND, PD, PSV, matrix correlation methods) can be used with presence and absence data, as well as those considering the species relative abundance (e.g., Cadotte et al. 2009). In general, most of the methods tend to reject the null hypothesis when it is true (high type I error) in cases with a spatial or phylogenetic autocorrelation in species abundance. Thus, appropriate null models preserving such autocorrelation should be used (Hardy 2008). For instance, a reliable null model is produced by permuting species with similar abundances across the tips of the phylogeny (Hardy 2008, Kembel 2009).

\section{Box 3. Evaluating the statistical performance of phylogenetic structure methods.}

The evaluation of the statistical performance requires that we simulate known phylogenetic community patterns, then apply the evaluated metric, and finally quantify the number of times that the metric can correctly detect the simulated pattern. It is known that the phylogenetic community structure is sensitive to the size of the regional and community species pool, tree imbalance and resolution, trait evolution, and the assembly process (Kraft et al. 2007, Hardy 2008, Kembel 2009, Swenson 2009). The simulation involves defining the phylogenetic tree of the regional species pool, simulating the trait evolution in this tree, and selecting the species assembling each community following one of the assembly rules (habitat filtering, random assembly, and species interaction). This process should be repeated many times to obtain the error rate.

To detect type I error, it is necessary to generate communities with no phylogenetic structure. This can be achieved by simulating a random trait evolution or a random community assembly. The former can be easily generated by randomly allocating trait values to the tips of the phylogeny of the regional pool. The latter can be produced by randomly allocating the species from the regional pool into the communities.

To detect type II error, it is necessary to generate communities with either clustered or overdispersed phylogenetic structures. To generate a clustered community, we need to mimic the conserved trait evolution and the habitat filtering process (Com-1 in figure 3). A conserved trait can be simulated using a Brownian motion model or by manually ordering trait values across the tips of the phylogeny; then habitat filtering may be applied by selecting species whose trait values fall within a reduced range (i.e., mimicking survival in the environment). Phylogenetic overdispersed communities can be simulated by generating conserved traits and then selecting dissimilar species in relation to this trait (Com-2 in figure 3), or by generating a convergent trait and selecting similar species (habitat filtering; Com-3 in figure 3). To select phenotypically dissimilar species requires first computation of the pair-wise Euclidean distances between all species in trait space, then selection the species pair with the smallest distance separating them; from that pair, we need to remove the species with the smallest mean distance to all other species in the community (Kraft et al. 2007). A convergent trait can be generated by assigning different trait values in a repeated pattern across the tips of the phylogeny.

Finally, the performance of the metric would be the proportion of runs detecting the simulated phylogenetic structure. 


\section{A step forward}

The current refinements in the methods for detecting phylogenetic and phenotypic community structure depart from the simplistic view of the dichotomy between competition and habitat filtering, and acknowledge the evolutionary dynamics in the community assembly processes.

\section{Going beyond the dichotomy of competition versus filtering.} Recently, the application of community phylogenetic methods has helped to demonstrate that competition is not the only force limiting the similarity of co-occurring species, and that abiotic factors are not the only filtering forces (Cavender-Bares et al. 2009). Researchers have suggested that pathogen-host interactions overdisperse the phylogenetic structure of forest communities, as closely related trees are similarly susceptible to infection (e.g., Gilbert and Webb 2007). Likewise, predation may produce phylogenetic clustering (Vamosi and Vamosi 2007) or overdispersion (Brower 1996). Of special relevance is the identification of positive interactions as forces assembling communities, in contrast to the traditional thinking that only negative interactions are relevant in structuring communities. Facilitation is an ecological interaction usually occurring between phylogenetically distant related species, and therefore communities driven by facilitation are phylogenetically overdispersed (e.g., Valiente-Banuet and Verdú 2007). Pollinator facilitation can lead to patterns of phylogenetic clustering or overdispersion, depending on whether pollinator traits are evolutionarily conserved (Sargent and Ackerly 2008). Further mutualistic interactions such as Müllerian mimicry can also shape community structure depending on the co-occurrence of the mimetic patterns (Elias et al. 2008).

Both limiting similarity and habitat filtering may occur in a given community (Weiher et al. 1998). Before species interactions limit the similarity of co-occurring species, environmental filtering may restrict the species composition of the community (Wilson 2007). Indeed, there is evidence of both niche differentiation (low SD of nearest-neighbor distance among trait values) and filtering (small range of observed traits) in different traits of the same community, or even for the same trait of a given community (Kraft et al. 2008, Cornwell and Ackerly 2009). Helmus and colleagues (2007a) provided a method combining the environment- and phylogeny-based approaches to simultaneously test whether the final composition of communities is the result of the combined effect of both competition and habitat filtering. As limiting similarity and environmental filtering lead to opposite phenotypic and phylogenetic patterns (figure 3), they may obscure each other's effects, precluding the detection of community structure unless they are explicitly considered separately (Helmus et al. 2007a). First, environmental filtering is detected by the phylogenetic signal of the regression coefficients of species occurrence with environment (see section on environment-based methods above, and 4 in figure 1). Then, limiting similarity is inferred by correlating phylogenetic and co-occurrence distances after statistically removing the environmental effects.
The possibility of more than one filter at local scale cannot be discounted (Keddy 1992). For instance, given that an environmental filter allows the persistence of closely related species, and that these species tend to share the same predators and parasites (Vamosi and Vamosi 2007), predation could well be a second filter acting on the previously filtered community.

The simultaneous action of two community assembly processes could also be detected by combining the convex hull volume approach with a test of limiting similarity within the community (Cornwell et al. 2006). Once the reduction of the community volume is demonstrated (habitat filtering), a second assembly process (limiting similarity) could be detected by comparing the spatial point pattern between species in the trait space with the null model (figure 4), such that trait overdispersion would be an indicator of limiting similarity (Ricklefs and Travis 1980, Weiher et al. 1998, Kraft et al. 2008). For instance, Pacific rockfish assemblages show overdispersion of traits related to trophic position and clustering of traits involved in depth adaptation (Ingram and Shurin 2009).

In conclusion, environmental filtering and different biotic interactions may be acting together to assemble the community, and we therefore need to leave behind the simplistic idea of two opposite assembly forces.

\section{Toward including evolutionary dynamics into community assembly.}

The community assembly theory has largely viewed communities as the outcome of ecological dynamics (mainly filtering and competition, but see above). The role of community dynamics in triggering diversification and trait divergence has received little attention (Johnson and Stinchcombe 2007). However, recent approaches aim to incorporate trait divergence (Pausas and Verdú 2008, Prinzing et al. 2008) and adaptive diversification (Fukami et al. 2007) into the framework of community assembly (Emerson and Gillespie 2008).

Trait divergence within a community can arise from species interactions. Competitive exclusion acts at the local scale, whereas evolutionary divergence of traits appears across the habitat species pool (Prinzing et al. 2008). Consequently, phenotypic overdispersion by trait divergence can be detected as a negative relationship between phylogenetic and phenotypic distances across the entire regional species pool (i.e., including different habitat types), and not within a given habitat (Prinzing et al. 2008). By applying this method, Prinzing and colleagues found evidence for evolutionary divergence of persistence (e.g., lifespan) and dispersal (e.g., seed weight) traits in plant communities, suggesting the importance of including evolutionary dynamics in community assembly theory.

Evolutionary diversification can certainly shape the community structure, but clear evidence of this process has been shown only in short-lived organisms. By experimentally modifying diversification rates in bacteria, Fukami and colleagues (2007) obtained communities with different phenotypic structures (different proportion of 


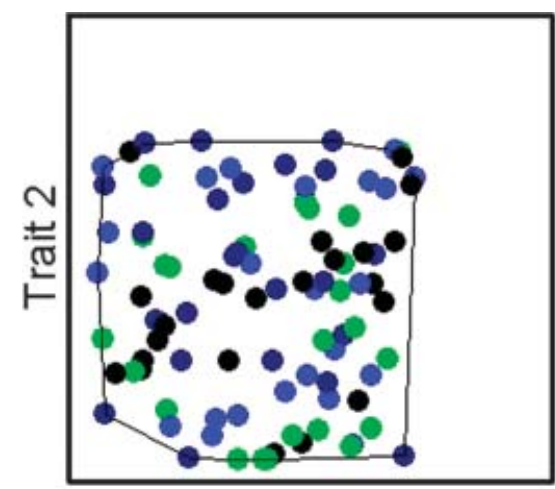

Trait 1

$\mathrm{NNsd}=0.03$

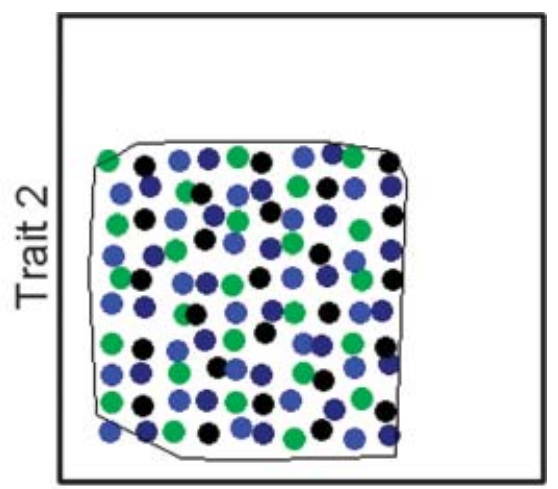

Trait 1

NNsd $=0.013$

Figure 4. The distribution of trait values within the reduced morphospace by habitat filtering (from figure 2c) may be random (left) and overdispersed (right; with lower standard deviation of the nearest-neighbor distance, NNsd). In the former case only one assembly process (filtering) is acting, whereas in the latter both filtering and limiting similarity are acting. The polygon indicates the convex hull.

diversification; on the other hand, a scenario with high beta diversity and high phylogenetic beta diversity indicates phylogenetic overdispersion driven by early diversifications.

Approaches that include both local and historic processes allow us to overcome the traditional dichotomy of the importance of local versus regional processes in assembling communities. We have shown that the new methodological advances in community ecology explicitly incorporate trait divergence, species diversification, and dispersal limitations, together with local processes, and thus we are on the way to establishing the desired bridge between community ecology and evolutionary biology (Johnson and Stinchcombe 2007, Cavender-Bares et al. 2009). bacteria morphotypes). Given that most traits are evolutionarily conserved (Blomberg et al. 2003), species arising after diversification in a particular clade will share similar trait states. This process would lead to an overrepresentation of the ancestral trait of that clade in the community, and consequently to phenotypic clustering (Pausas and Verdú 2008). Indeed, several diversification scenarios of random branching processes, such as pure birth or birthdeath models, can generate phenotypic clustering. To test whether the phenotypic structure of communities is mediated by an adaptive diversification of a particular clade, we need, as suggested by Pausas and Verdú (2008), to (a) test whether the branching pattern of the phylogeny fits to one of above mentioned branching scenarios (e.g., by means of a likelihood ratio test; Rabosky 2006), and (b) compare the morphospace occupied by the community with a null morphospace computed by simulating the evolution of traits based on the observed trait variance-covariance matrix (Revell et al. 2007). If the observed morphospace is not different from the morphospace obtained with simulated trait data, then we cannot reject the possibility that the phenotypic community structure is mediated by an adaptive diversification.

In a biogeographical context, trait evolution and species diversification can be mediated by migration and dispersal processes (e.g., Moen et al. 2009). Recent approaches that work by comparing beta diversity (species turnover across the space) with phylogenetic beta diversity (i.e., species turnover including the temporal dimension defined by phylogenetic distances) allow the differentiation of the relative role of regional processes, including trait evolution and species diversification into the assembly of communities (Graham and Fine 2008). For example, high beta diversity combined with low phylogenetic beta diversity indicates a phylogenetic clustering mediated by recent

\section{Future directions}

Despite the apparent jungle of terms and methods, here we show that a unified framework involving the relationships between four concepts (co-occurrence, phenotype, phylogeny, and environment) is sufficient to obtain an integrated view of the processes structuring communities. A checklist integrating the concepts proposed above (figure 1) as well as the software available (table 1) may guide researchers performing a complete community structure study (box 4). The field of community ecology may benefit from new theoretical and methodological advances that would provide the opportunity to deal with future challenges in conservation biology. Below we outline these possible advances.

From a theoretical point of view, the field may benefit from current advances in the theory of species coexistence and in the theory of ecological networks. For instance, there is growing evidence that coexistence can be driven by the long-term rates at which community members recover from low density by means of equalizing and stabilizing mechanisms; equalizing effects minimize fitness differences between species, whereas stabilizing processes increase the negative intraspecific interactions (Chesson 2000, Holt 2006). Similarly, network theory is providing clues for understanding species coexistence (Chesson and Kuang 2008); for example, network parameters such as nestedness can shed light on the strength of interspecific competition and species coexistence (Bastolla et al. 2009).

Methodological advances in understanding community structure are associated with the increasing availability of databases of both phylogenetic and phenotypic information. Such growth of phylogenetic information would ideally allow us to move from phylogenetics to phylogenomics and achieve not only a better understanding of the historical relationships between species but also infer the genetic signature of both ecological (e.g., filtering) and evolutionary 


\section{Box 4. A recipe for the ideal study of community structure.}

Below we provide the five key steps to understanding the processes structuring community composition. Full details and references are given in the main text and in the corresponding figures and boxes.

1. Define the geographical scale and taxonomical scope.

2. Identify the four corners (figure 1).

- Identify and measure the environmental factors determining the species composition (environment).

- Identify and measure the set of traits conferring survival in the given environment (phenotypes).

- Sample species composition (co-occurrence).

- Obtain a well-resolved phylogeny (phylogeny).

3. Identify the processes shaping the phenotypic structure.

- Test whether species with similar phenotypes co-occur in the same environment.

- Test for phenotypic structure (figure 2): Phenotypic clustering suggests that habitat filtering is a dominant process. Phenotypic overdispersion suggests that species interactions (e.g., competition, facilitation) are good candidates to be responsible for community structure. In that case, one would have to test the assumption that the outcome of the interaction is the result of phenotypic similarity among involved species (e.g., competition is stronger among similar species; facilitation is stronger among dissimilar species). Note that both clustering (reduced range) and overdispersion (even spacing) of traits may occur (see figure 4).

4. Test whether the phenotypic structure determines the phylogenetic structure of the community (figure 3).

- Test whether co-occurring species are more or less related than predicted by chance (phylogenetic structure; see box 2).

- Test whether closely related species resemble each other more or less than predicted by chance (i.e., trait evolution; the species response to environment can also be considered as a trait).

- The combination of trait evolution and phylogenetic structure will allow us to quantify the relative contribution of community structure processes (figure 3).

5. Evaluate the statistical power of the analysis (box 3 ).

processes (e.g., diversification). Furthermore, there is a need to improve the characterization of phenotypes by considering individual variability and traits more proximal to biological processes than the classical surrogates (e.g., direct information of growth rate instead of wood density). The difficulty of measuring some functional traits relevant to community structure (e.g., allelopathic effects) may be overcome by working with functional genes. For example, microarray-based genomic technology is allowing the detection of functional genes responsible for processes that structure microbial communities and ecosystem function. The barrage of genetic and phenotypic data may overwhelm the current statistical methods. We urgently need to integrate this huge amount of information and the associated uncertainty of the resulting phylogenetic trees (e.g., topology, branch length) and phenotypic characterizations (e.g., intraspecific trait variability, ancestral reconstruction models). Although some advances have been made in comparative methods (e.g., Bayesian statistics), little is done in that direction in the framework of community ecology.

We also need to move toward experimental approaches in order to avoid the myriad confounding factors affecting natural communities. This would also allow us to rigorously validate the predictive value of the methods used to infer assembly processes. Experimentation may imply modification of the biotic and abiotic factors responsible of assembly processes, as well as the species pool. For instance, modification of biotic factors is possible by removing competitors, adding facilitators or predators, and so on. Abiotic factors can also be experimentally altered by shading, burning, irrigating, fertilizing, or using open-top chambers or free-air carbon enrichmnent experiments. The maximum control of factors, including the species pool, can be achieved by micro- and mesocosm experiments. However, it is worth noting that the greater the control, the lower the realism.

The array of methods described here may contribute to better planning of conservation policies. Given that most traits are evolutionarily conserved, long-distantly related species are those that capture the highest morphological and functional variability. Consequently, maximizing phylogenetic diversity better captures evolutionary information than species richness, and thus it has been shown as the best bet hedging conservation strategy (Forest et al. 2007). Restoration ecology may also benefit from the recent findings on community ecology. For instance, the phylogenetic-based approach can optimize the recent restoration practices based on facilitation by considering that facilitation tends to occur between distantly related species (Valiente-Banuet and Verdú 2007). Similarly, the phenotypebased approach provides a framework to protect successful restoration efforts against invasion by selecting native species with traits similar to likely invaders and maximizing trait variability (Funk et al. 2008). In addition, the environment-based approach may provide insights on the management of ecological filters (nutrients, fire, mowing); for instance, managing fire regimes may select for or against desired and undesired species (Funk et al. 2008). 
A promising direction of the methods reviewed here is to predict ecosystem function on the basis of phylogenetic relatedness. For instance, Cadotte and colleagues (2008) showed that plant community biomass is better predicted by considering species relatedness of the community than by the traditional biodiversity indicators such as the number of species or functional groups. Phylogenetic relatedness can also effectively act as a proxy for species' responses to disturbance, and thus these methods can be applied to temporal slices to detect changes in phylogenetic and phenotypic structure (Helmus et al. 2010). Linking phylogenetics and ecosystem function opens up the possibility of predicting ecological consequences of biodiversity shifts in a changing world. Indeed, phylogenetics can guide better taxon sampling of key physiological traits for scaling from organism physiology to global processes (Edwards et al. 2007).

\section{Acknowledgments}

We thank María Clara Castellanos, Steve Kembel, Evan Weiher, and three anonymous referees for helpful comments and suggestions. This work has been developed under the framework of the Spanish projects VIRRA (CGL200912048/BOS) and VAMPIRO (CGL2008-05289-C02-01).

\section{References cited}

Abrams P. 1983. The theory of limiting similarity. Annual Review of Ecology and Systematics 14: 359-376.

Bastolla U, Fortuna MA, Pascual-Garcia A, Ferrera A, Luque B, Bascompte J. 2009. The architecture of mutualistic networks minimizes competition and increases biodiversity. Nature 458: 1018-1020.

Blomberg SP, Garland T, Ives AR. 2003. Testing for phylogenetic signal in comparative data: Behavioral traits are more labile. Evolution 57: 717-745.

Brower AVZ. 1996. Parallel race formation and the evolution of mimicry in Heliconius butterflies: A phylogenetic hypothesis from mitochondrial DNA sequences. Evolution 50: 195-221.

Cadotte MW, Cardinale BJ, Oakley TH. 2008. Evolutionary history and the effect of biodiversity on plant productivity. Proceedings of the National Academy of Sciences 105: 17012-17017.

Cadotte MW, Hamilton MA, Murray BR. 2009. Phylogenetic relatedness and plant invader success across two spatial scales. Diversity and Distributions 15: 481-488.

Cavender-Bares J, Ackerly DD, Baum DA, Bazzaz FA. 2004. Phylogenetic overdispersion in Floridian oak communities. American Naturalist 163: 823-843.

Cavender-Bares J, Keen A, Miles B. 2006. Phylogenetic structure of Floridian plant communities depends on taxonomic and spatial scale. Ecology 87: S109-S122.

Cavender-Bares J, Kozak KH, Fine PVA, Kembel SW. 2009. The merging of community ecology and phylogenetic biology. Ecology Letters 12 : 693-715.

Chesson P. 2000. Mechanisms of maintenance of species diversity. Annual Review of Ecology and Systematics 31: 343-366.

Chesson P, Kuang JJ. 2008. The interaction between predation and competition. Nature 456: 235-238.

Coca M, Pausas JG. 2009. Regeneration traits are structuring phylogenetic diversity in cork oak (Quercus suber) woodlands. Journal of Vegetation Science 20: 1009-1015.

Connor EF, Simberloff D. 1979. The assembly of species communities: Chance or competition? Ecology 60: 1132-1140.

Connor EF, Hosfield E, Meeter DA, Niu XF. 1997. Tests for aggregation and size-based sample-unit selection when sample units vary in size. Ecology 78: $1238-1249$.
Cornwell WK, Ackerly DD. 2009. Community assembly and shifts in plant trait distributions across an environmental gradient in coastal California. Ecological Monographs 79: 109-126.

Cornwell WK, Schwilk DW, Ackerly DD. 2006. A trait-based test for habitat filtering: Convex hull volume. Ecology 87: 1465-1471.

Darwin CR. 1859. On the Origin of Species by Means of Natural Selection, or the Preservation of Favoured Races in the Struggle for Life. John Murray.

Diamond JM, Cody ML. 1975. Assembly of species communities. Pages 342-444 in Diamond JM, Cody ML, eds. Ecology and Evolution of Communities. Harvard University Press.

Dray S, Legendre P. 2008. Testing the species traits-environment relationships: The fourth-corner problem revisited. Ecology 89: 3400-3412.

Edwards EJ, Still CJ, Donoghue MJ. 2007. The relevance of phylogeny to studies of global change. Trends in Ecology and Evolution 22: 243-249.

Elias M, Gompert Z, Jiggins C, Willmott K. 2008. Mutualistic interactions drive ecological niche convergence in a diverse butterfly community. PLoS Biology 6: 2642-2649.

Emerson BC, Gillespie RG. 2008. Phylogenetic analysis of community assembly and structure over space and time. Trends in Ecology and Evolution 23: 619-630.

Faith DP. 1992. Conservation evaluation and phylogenetic diversity. Biological Conservation 61: 1-10.

Felsenstein J. 2008. Comparative methods with sampling error and withinspecies variation: Contrasts revisited and revised. American Naturalist 171: 713-725.

Ferrier S, Manion G, Elith J, Richardson K. 2007. Using generalized dissimilarity modelling to analyse and predict patterns of beta diversity in regional biodiversity assessment. Diversity and Distributions 13: 252-264.

Forest F, Grenyer R, Rouget M, Davies TJ, Cowling RM, Faith DP, Balmford A, Manning JC, Proches S, van der Bank M. 2007. Preserving the evolutionary potential of floras in biodiversity hotspots. Nature 445 : $757-760$.

Fukami T, Beaumont HJE, Zhang X-X, Rainey PB. 2007. Immigration history controls diversification in experimental adaptive radiation. Nature 446: 436-439.

Funk JL, Cleland EE, Suding KN, Zavaleta ES. 2008. Restoration through reassembly: Plant traits and invasion resistance. Trends in Ecology and Evolution 23: 695-703.

Gilbert GS, Webb CO. 2007. Phylogenetic signal in plant pathogen-host range. Proceedings of the National Academy of Sciences 104: 49794983.

Gotelli NJ, Graves GR. 1996. Null Models in Ecology. Smithsonian Institute Press.

Graham CH, Fine PVA. 2008. Phylogenetic beta diversity: Linking ecological and evolutionary processes across space in time. Ecology Letters 11: $1265-1277$.

Hardy OJ. 2008. Testing the spatial phylogenetic structure of local communities: Statistical performances of different null models and test statistics on a locally neutral community. Journal of Ecology 96: 914-926.

Hardy OJ, Senterre B. 2007. Characterizing the phylogenetic structure of communities by an additive partitioning of phylogenetic diversity. Journal of Ecology 95: 493-506.

Helmus MR, Savage K, Diebel MW, Maxted JT, Ives AR. 2007a. Separating the determinants of phylogenetic community structure. Ecology Letters 10: 917-925.

Helmus MR, Bland TJ, Williams CK, Ives AR. 2007b. Phylogenetic measures of biodiversity. American Naturalist 169: E68-E83.

Helmus MR, Keller W, Paterson MJ, Yan ND, Cannon CH, Rusak JA. 2010. Communities contain closely related species during ecosystem disturbance. Ecology Letters 13: 162-174.

Holt RD. 2006. Emergent neutrality. Trends in Ecology and Evolution 21: 531-533.

Huisman J, Olff H, Fresco LFM. 1993. A hierarchical set of models for species response analysis. Journal of Vegetation Science 4: 37-46. 
Hutchinson GE. 1957. Population studies: Animal ecology and demography. Concluding remarks. Cold Spring Harbor Symposia on Quantitative Biology 22: 415-427.

Ingram T, Shurin JB. 2009. Trait-based assembly and phylogenetic structure in northeast Pacific rockfish assemblages. Ecology 90: 2444-2453.

Johnson MTJ, Stinchcombe JR. 2007. An emerging synthesis between community ecology and evolutionary biology. Trends in Ecology and Evolution 22: 250-257.

Keddy PA. 1992. Assembly and response rules: Two goals for predictive community ecology. Journal of Vegetation Science 3: 157-164.

Kembel SW. 2009. Disentangling niche and neutral influences on community assembly: Assessing the performance of community phylogenetic structure tests. Ecology Letters 12: 949-960.

Kraft NJB, Cornwell WK, Webb CO, Ackerly DD. 2007. Trait evolution, community assembly, and the phylogenetic structure of ecological communities. American Naturalist 170: 271-283.

Kraft NJB, Valencia R, Ackerly DD. 2008. Functional traits and nichebased tree community assembly in an Amazonian forest. Science 322: 580-582.

Leibold MA, et al. 2004. The metacommunity concept: A framework for multi-scale community ecology. Ecology Letters 7: 601-613.

MacArthur R, Levins R. 1967. The limiting similarity, convergence, and divergence of coexisting species. American Naturalist 101: 377.

Mayfield MM, Boni MF, Ackerly DD. 2009. Traits, habitats, and clades: Identifying traits of potential importance to environmental filtering. American Naturalist 174: E1-E22.

Moen DS, Wiens JJ, Pfennig D. 2009. Phylogenetic evidence for competitively driven divergence: Body-size evolution in Caribbean treefrogs (Hylidae: Osteopilus). Evolution 63: 195-214.

Noble IR, Slatyer RO. 1980. The use of vital attributes to predict successional changes in plant communities subject to recurrent disturbances. Vegetatio 43: 5-21.

Ojeda F, Pausas JG, Verdú M. 2010. Soil shapes community structure through fire. Oecologia 163: 729-735. doi:10.1007/s00442-009-1550-3

Pausas JG, Verdú M. 2008. Fire reduces morphospace occupation in plant communities. Ecology 89: 2181-2186.

Pavoine S, Love MS, Bonsall MB. 2009. Hierarchical partitioning of evolutionary and ecological patterns in the organization of phylogenetically structured species assemblages: Application to rockfish (genus: Sebastes) in the southern California bight. Ecology Letters 12: 898-908.

Perry JN, Liebhold AM, Rosenberg MS, Dungan J, Miriti M, Jakomulska A Citron-Pousty S. 2002. Illustrations and guidelines for selecting statistical methods for quantifying spatial pattern in ecological data. Ecography 25: 578-600.

Pillar VD, Duarte LdS. 2010. A framework for metacommunity analysis of phylogenetic structure. Ecology Letters 13: 587-596.

Prinzing A, Reiffers R, Braakhekke WG, Hennekens SM, Tackenberg O, Ozinga WA, Schaminee JHJ, Groenendael JMV. 2008. Less lineagesmore trait variation: Phylogenetically clustered plant communities are functionally more diverse. Ecology Letters 11: 809-819.

Proches S, Wilson JRU, Cowling RM. 2006. How much evolutionary history in a $10 \times 10 \mathrm{~m}$ plot? Proceedings of the Royal Society B 273 : $1143-1143$.

Rabosky DL. 2006. LASER: A maximum likelihood toolkit for detecting temporal shifts in diversification rates from molecular phylogenies. Evolutionary Bioinformatics Online 2006: 257-260.

Rabosky DL, Reid J, Cowan M, Foulkes J. 2007. Overdispersion of body size in Australian desert lizard communities at local scales only: No evidence for the Narcissus effect. Oecologia 154: 561-570.

Revell LJ, Harmon LJ, Langerhans RB, Kolbe JJ. 2007. A phylogenetic approach to determining the importance of constraint on phenotypic evolution in the neotropical lizard Anolis cristatellus. Evolutionary Ecology Research 9: 261-282.

Revell LJ, Harmon LJ, Collar DC. 2008. Phylogenetic signal, evolutionary process, and rate. Systematic Biology 57: 591-601.

Ricklefs RE, Travis J. 1980. A morphological approach to the study of avian community organization. The Auk 97: 321-338.

Sargent RD, Ackerly DD. 2008. Plant-pollinator interactions and the assembly of plant communities. Trends in Ecology and Evolution 23: $123-130$.

Simberloff DS. 1970. Taxonomic Diversity of Island Biotas. Evolution 24: $23-47$.

Stayton CT. 2008. Is convergence surprising? An examination of the frequency of convergence in simulated datasets. Journal of Theoretical Biology 252: 1-14.

Swenson NG. 2009. Phylogenetic resolution and quantifying the phylogenetic diversity and dispersion of communities. PLoS ONE 4: e4390-e4390.

Swenson NG, Enquist BJ, Pither J, Thompson J, Zimmerman JK. 2006. The problem and promise of scale dependency in community phylogenetics. Ecology 87: 2418-2424.

Tilman D. 1985. The resource-ratio hypothesis of plant succession. American Naturalist 125: 827-852.

Ulrich W. 2004. Species co-occurrences and neutral models: Reassessing J. M. Diamond's assembly rules. Oikos 107: 603-609.

Valiente-Banuet A, Verdú M. 2007. Facilitation can increase the phylogenetic diversity of plant communities. Ecology Letters 10: 10291036.

Vamosi JC, Vamosi SM. 2007. Body size, rarity, and phylogenetic community structure: Insights from diving beetle assemblages of Alberta. Diversity and Distributions 13: 1-10.

Vamosi SM, Heard SB, Vamosi JC, Webb CO. 2009. Emerging patterns in the comparative analysis of phylogenetic community structure. Molecular Ecology 18: 572-592.

van der Valk AG. 1981. Succession in wetlands: A Gleasonian approach. Ecology 62: 688-696.

Verdú M, Pausas JG. 2007. Fire drives phylogenetic clustering in Mediterranean basin woody plant communities. Journal of Ecology 95: $1316-1323$.

Webb CO, Ackerly DD, McPeek MA, Donoghue MJ. 2002. Phylogenies and community ecology. Annual Review of Ecology and Systematics 33: 475-505.

Weiher E, Keddy PA. 1995. The assembly of experimental wetland plant communities. Oikos: 323-335.

. 1999. Ecological Assembly Rules: Perspectives, Advances, Retreats. Cambridge University Press.

Weiher E, Clarke GDP, Keddy PA. 1998. Community assembly rules, morphological dispersion, and the coexistence of plant species. Oikos 81: 309-322.

Wilson JB, Weiher E, Keddy P. 1999. Assembly rules in plant communities. Pages 130-164. Ecological Assembly Rules: Perspectives, Advances, Retreats. Cambridge University Press.

Wilson JB. 2007. Trait-divergence assembly rules have been demonstrated: Limiting similarity lives! A reply to Grime. Journal of Vegetation Science 18: $451-452$

Juli G. Pausas (juli.g.pausas@uv.es) and Miguel Verdú (miguel.verdu@uv.es) are ecologists at the Centro de Investigaciones sobre Desertificacion of the Spanish National Research Council, in Valencia (CIDE, CSIC), Spain. Both are interested in how plant species and communities are shaped by different ecological and evolutionary forces. 